\title{
An easy to make and simple designed rearing apparatus for Chironomidae
}

\author{
Armin Namayandeh ${ }^{1}$ and David V. Beresford ${ }^{2}$ \\ ${ }^{1}$ Environmental \& Life Sciences, Environmental Science Building Room A 105 Trent University, Peterbor- \\ ough, Ontario, Canada, K9J 7B8, Tel: (705)748-1011 X 7891 \\ E-mail: arminnamayand@trentu.ca \\ ${ }^{2}$ Biology Department, Trent University, LHS D21, K9J 7B8, (705)748-1011 X 7540 Peterborough, On- \\ tario, Canada \\ E-mail: davidberesford@,trentu.ca
}

\section{Introduction}

Identification of immature Chironomidae can be impossible, as most species are identified based on adult males, for which the associated pupae or larvae are not known or described. To overcome this, Chironomidae researchers rear larvae and pupae in aquaria to obtain the adults. This is usually done on fourth or final instar larvae collected from aquatic habitats, and mass reared (e.g. Biever 1965; Hein and Mahadeva 1993). An advantage of mass rearing is that a large number of specimens can be obtained for identification. However, with mass rearing, matching individual larvae with pupal and adult stages is not possible. To overcome this, larvae can be grown individually, a potentially costly undertaking. Even though most chironomids can withstand the room temperatures, most cannot tolerate low oxygen levels that are created in the vials (Mendes 2002).

In this paper we describe an inexpensive and rapid method of constructing an apparatus so that all three stages of individual specimen can be obtained, and that maintains sufficient oxygen during the rearing process.

\section{Materials and Methods}

Rearing vials were placed in a modified base constructed from centrifuge tube stands made of styrofoam, which are readily available in chemistry or biology labs. A standard foam is $20 \mathrm{~cm}$ long, $16 \mathrm{~cm}$ wide and $3 \mathrm{~cm}$ indeep, and has 25 holes for individual centrifuge tubes. To make the base we cut a foam stand in half to produce a $10 \mathrm{~cm}$ by $16 \mathrm{~cm}$ stand, and glued this to a second stand to create a stepped structure (Figure 1i).

The rearing chamber was constructed from two centrifuge tubes to create an angled chamber with a screw cap on each end. The bottom end with screw cap was made water tight using plumbers tape and further sealed from the outside of the screw cap using aquarium glue. The top cap was left removable to allow access, and removal of emergent adults.

To modify the tubes, the bottom tube was cut perpendicularly at the $7.5 \mathrm{~cm}$ height (Figure 1ii). The bottom section had two drilled holes, one sized to receive an air tube that feds the centrifuge tube from an air pump, and the second used to feed the larvae. We found that the second hole could be dispensed with, and reared $4^{\text {th }}$ instar larvae successfully in using stream water with no feeding access port. When the second hole was installed, it was outfitted with a small plastic tube, $1.5 \mathrm{~cm}$ in length to allow feeding (Figure 1iii). The top section was cut diagonally at the tip at a $45^{\circ}$ angle (Figure 1ii). The bottom vial was then inserted into the top at a $135^{\circ}$ angle (Figure 1iii) and the joint of these two sealed using aquarium silicon. Finally, both the feeding tube and the air tube were made watertight using aquarium cement.

Using this apparatus, stream water and individual larvae were added to the rearing chamber from the top, and the chamber secured to the base using tape. The bottom fitted the stand holes and was secure.

Because the apparatus is small, any air pressure entering needed to be low to prevent water from splashing in the chamber. We adjusted the air pressure using hose clamps, and found that 1-2 air bubbles every second provided sufficient aeration. 

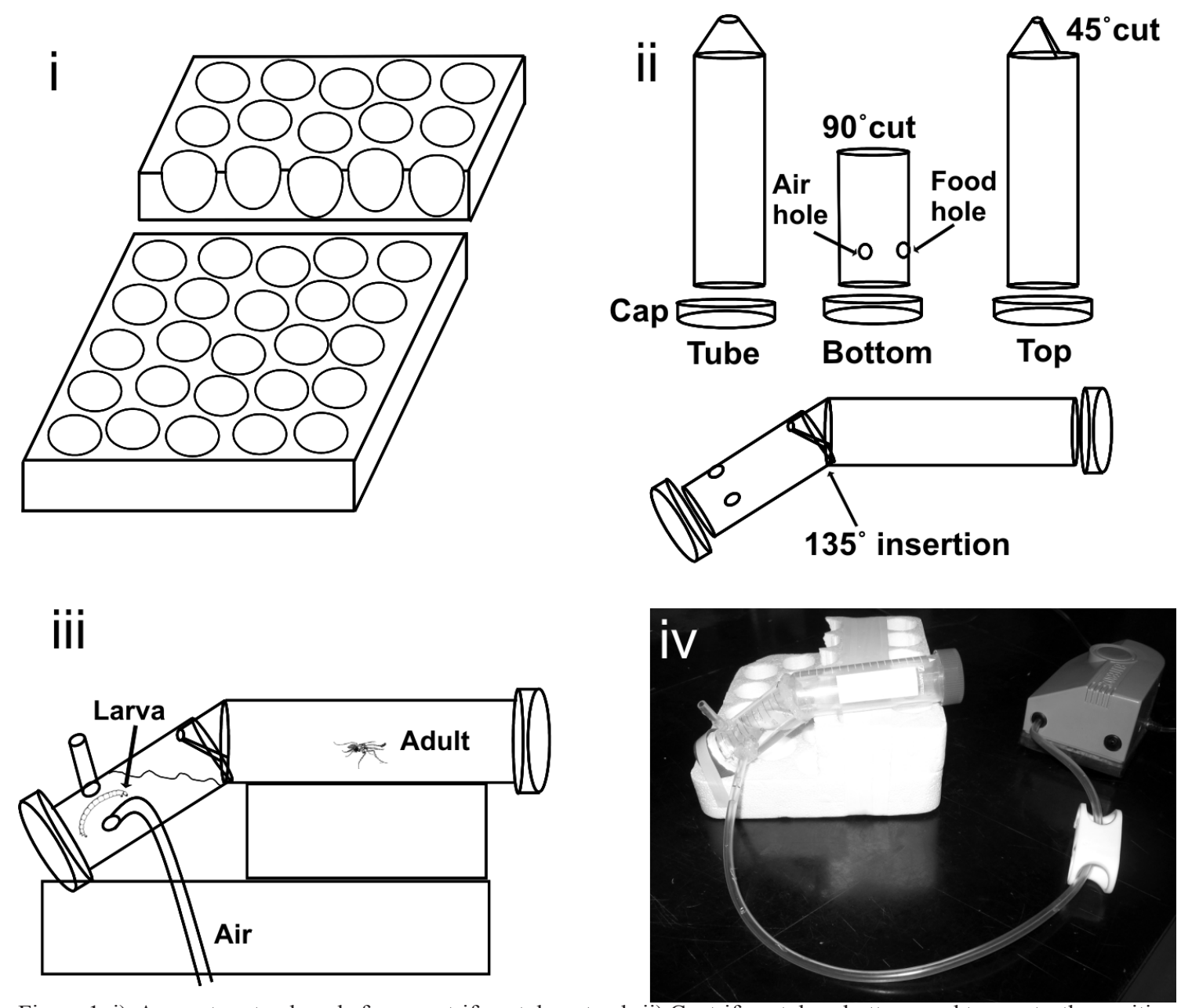

Figure 1: i) Apparatus stand made from centrifuge tubes stands ii) Centrifuge tubes, bottom and top parts, the position of holes and cuts, and assembling the top part to the bottom part iii) Apparatus on stand, position of larvae and adults iv) The apparatus assembled.

The larvae were placed at the base of chamber, and adults emerged into the top section, and remained dry due to the lip. Pupae exuviae and larva head capsule were obtained from the bottom part once the adults were removed (Figure 1iii). Using this apparatus we have been able to acquire all three stages of Chironomidae for identification.

\section{References}

Biever, K.D., 1965. A Rearing Technique for the Colonization of Chironomid Midges. - Annals of the Entomological Society of America 58: 135-136.

Hein, J. and Mahadeva, M.N., 1992. Rearing and Maintaining Midge Cultures (Chironomus tentans) for Laboratory Studies. - The American Biology Teacher 54: 236-240.

Mendes, H.F. 2002. Rearing Tanypodinae, Telmatogetoninae and Orthocladiinae in Brazil - an empirical approach. - Chironomus Newsletter on Chironomidae Research 15: 29-32. 